\title{
Persea americana L. (Avocado) Fruit Mesocarp intake in Experimental Diabetic Rats: Impacts and Implication of Mode of Consumption
}

\author{
Magnus Michael Chukwudike Anyakudo* and Ifeoluwa Adekunle Adediji \\ Endocrinology, Metabolism and Clinical Nutrition Research Unit, Department of Physiology, Faculty of Basic Medical Sciences, University of \\ Medical Sciences, Ondo City, Nigeria,
}

*Corresponding Author: Magnus Michael Chukwudike Anyakudo, Endocrinology, Metabolism and Clinical Nutrition Research Unit, Department of Physiology, Faculty of Basic Medical Sciences, University of Medical Sciences, P.M.B 536, Laje Road, Ondo City, Ondo State, Nigeria.

Received date: October 22, 2021; Accepted date: November 03, 2021; Published date: November 13, 2021

Citation: Magnus M.C. Anyakudo, Ifeoluwa A. Adediji, (2021). Persea americana L. (Avocado) Fruit Mesocarp intake in Experimental Diabetic Rats: Impacts and Implication of Mode of Consumption. J. Nutrition and Food Processing, 4(7); DOI:10.31579/2637-8914/071

Copyright: () 2021 Magnus Michael Chukwudike Anyakudo, This is an open access article distributed under the Creative Commons Attribution License, which permits unrestricted use, distribution, and reproduction in any medium, provided the original work is properly cited.

\begin{abstract}
Background: Few epidemiological data exist on the effects of the mode of consumption of avocado on diet quality, weight management, and lipoglycemic profile in diabetic condition and other metabolic disease risk factors.

Objectives: This study investigated the metabolic, lipoglycemic, and anthropometric impacts of avocado fruit mesocarp intake and the implication of its mode of consumption on body weight gain, lipid profile, glycemic tolerance and control in male diabetic Wistar rats.

Method: Twenty one (21) adult male Wistar rats (150-220g) were randomly categorized into three experimental groups $(\mathrm{n}=7$, each): Diabetic control fed with normal diet (DC); Diabetic rats fed with avocado supplemented diet (DSA); Diabetic rats treated with aqueous mesocarp extract of avocado (DAE). Diabetes was inducted with $150 \mathrm{mg} / \mathrm{dL}$, alloxan monohydrate solution intraperitoneally. Animals were fed according to the experimental design with water ad libitum for six weeks. Body weights and fasting blood glucose (FBG) concentrations were measured twice weekly. LP and OGTT were conducted. Microsoft Excel and statistical SPSS program version 22 were used for data analysis. Results are expressed as mean \pm SEM. Comparison between groups were made using Students't-test and one way ANOVA.
\end{abstract}

Results: Consumption of avocado mesocarp caused significant reduction in mean body weight gain (DSA: 13.75\%; DAE: $10.17 \% ; P$ value $<0.05)$ and blood glucose concentrations (DSA: $9.48 \%$; DAE: $21.0 \% ; P=0.002$ ) with significant improvement in glycemic tolerance and lipid profile (DAE > DSA) over the control. Peaked glycemic responses occurred at 30 minutes of glucose challenge in DSA and DAE groups.

Conclusion: Avocado fruit mesocarp intake reduced body weight gain and blood sugar with improved lipid profile and glycemic tolerance in experimental diabetic rats while the mode of consumption influenced its potential impacts.

Key words: avocado; body weight gain; diabetic rats; glycemic tolerance; lipid profile; persea americana; lipoglycemic; antiobesity; antidiabetic; antilipaemic potentials

\section{Introduction}

The medicinal relevance and the potential health benefits of avocado pear in the treatment of diverse disease conditions have been reported. However, a few epidemiological data exist on the effects of the mode of consumption of avocado on diet quality, weight management, and lipoglycemic profile in diabetic and other metabolic disease conditions. Persea americana commonly known as the "Avocado pear",
"Alligator pear", or Mexican avocado is a fruit tree whose various parts contain a variety of essential nutrients and important phytochemicals [15] contributing to its reported enormous potential health benefits. Diets rich in monounsaturated fats like those in avocado may reduce the risk of developing type 2 diabetes [6] while in those suffering from type 2 diabetes, monounsaturated fat diets have been reported to decrease fasting blood glucose [7], glycosylated haemoglobin (HbA1c) [8], fasting insulin 
with improved insulin sensitivity [9] and postprandial insulin response [10]. Avocado contains a unique sugar (D-manno-heptulose) that may help blood glucose control by reducing glycolysis without energy contribution [11,12]. While some studies [13-15] using healthy animals and human subjects revealed that avocado impacts positive benefits on weight gain, cholesterol, blood sugar levels and, insulin sensitivity, other studies [16-18] however, reported mixed findings. Observational studies revealed that mode of consumption of avocado vary in individuals influenced by various factors including culture, custom, environment, season, choice, traditions, and beliefs. Whether the mode of preparation and consumption of this fruit has effect or not on its potential benefits was a point of attraction investigated in this nutritional study along with the determination of its effect on body weight gain, lipid profile, glycemic tolerance and control. In this study, avocado fruit mesocarp was prepared and processed in two forms (aqueous extract and diet-supplemented forms) for consumption by the diabetic rats. In recent times, nutritional studies have gained a strong focus in proffering solutions to most dietrelated disorders. However, more studies are required to provide adequate epidemiological data on diet quality, weight management, and lipoglycemic profile in diabetic and other metabolic disease conditions. This experimentally-controlled nutritional study therefore, determined the metabolic, lipoglycemic, and anthropometric impacts of avocado fruit mesocarp prepared and consumed in two different forms in alloxan- induced diabetic rats with the rationale to provide rational basis for dietary selection, advice and recommendations where necessary, for optimal benefits and, therapeutic effects.

\section{Materials and Methods}

\section{Preparation of Aqueous Extract of Persea americana}

The ripe and unsoftened avocado pear purchased from our local market was washed and properly cleaned with distilled water. The outer green thin layer (exocarp) was peeled and discarded to expose the underlying mesocarp which was cut and weighed. $200 \mathrm{~g}$ of the peeled mesocarp was homogenized with $400 \mathrm{ml}$ of distilled water to a fine and smooth texture form using a laboratory blender. The aqueous mixture of avocado mesocarp was weighed and stored in refrigerator at $4^{\circ} \mathrm{C}$. Fresh portion of the mesocarp extract was prepared each week for oral administration.

\section{Test Feed and Composition}

The composition of the control and avocado-supplemented diets in this study was prepared based upon the standard diet formulas used to assess weight gain in rodents during commercial feeding studies. Table 1 shows the composition of the control and test diets.

\begin{tabular}{|l|l|l|l|}
\hline $\begin{array}{l}\text { NUTRIENT } \\
\text { COMPONENTS }\end{array}$ & INGREDIENTS USED & $\begin{array}{l}\text { NORMAL DIET } \\
\text { (CONTROL) } \\
\text { (\% per 100g of feed })\end{array}$ & $\begin{array}{l}\text { AVOCADO- } \\
\text { SUPPLEMENTED } \\
\text { (TEST) DIET } \\
(\% \text { per 100g of feed })\end{array}$ \\
\hline Carbohydrates & $\begin{array}{l}\text { Maize } \\
\text { Wheat offal }\end{array}$ & $\begin{array}{l}40 \% \\
15 \%\end{array}$ & $\begin{array}{l}40 \% \\
15 \%\end{array}$ \\
\hline Fat and Oil & $\begin{array}{l}\text { Palm kernel cake } \\
\text { Groundnut cake } \\
\text { Soya bean meal }\end{array}$ & $20 \%$ & $20 \%$ \\
& $\begin{array}{l}10 \% \\
10.5 \%\end{array}$ & $\begin{array}{l}10 \% \\
10.5 \%\end{array}$ \\
\hline Protein & Oyster shell & $1.0 \%$ & $1.0 \%$ \\
\hline Vitamins & Growth premix & $3.0 \%$ & $0.15 \%$ \\
\hline Mineral Salt & Salt & $0.25 \%$ & $0.25 \%$ \\
\hline ADDITIVES & Avocado & - & $0.1 \%$ \\
\hline & TOTAL & $100 \%$ & $100 \%$ \\
\hline
\end{tabular}

Table 1: Percentage Composition of Control and Test Diets

\section{Experimental Animals and Diets}

Twenty-one male Wistar rats (Rattus norvegicus) weighing above $150 \mathrm{~g}$ were obtained from the disease-free stock of a farm in Ife, Osun state, Nigeria. The animals were kept in polypropylene cages with stainless wire mesh top in a well-ventilated animal house maintained at normal and standard laboratory conditions of temperature and relative humidity for two weeks to allow them acclimatize to the environment. During this period, they were fed with commercially available standard rat feed and water ad libitum. Replaceable numbered blotters papers were placed under each cage to catch the spilled diet that was measured to make up for the daily serving ration. The rats were weighed twice weekly to ensure that no rat outside the range of $160-200 \mathrm{~g}$ was used.

\section{Induction of Diabetes}

Following the period of acclimatization, all the rats were fasted overnight (15 hours) before diabetes induction which was achieved with freshly prepared alloxan monohydrate (Kermel chemicals, China) dissolved in sterile normal saline and administered intraperitoneally at a dose of $150 \mathrm{mg} / \mathrm{kg}$ body weight. Diabetes was confirmed four days later using a glucometer (Fine Test Blood Glucose Monitoring System, OSANG Healthcare Co., Ltd., Korea). Rats with fasting blood sugar (FBS) level above $150 \mathrm{mg} / \mathrm{dL}$ were selected and grouped into three experimental groups according to the experimental design.

\section{Experimental Design}

The animals were categorized into three groups $(n=7$, each group) after induction as follows:

Group DC: Diabetic rats fed with normal diet (Control)

Group DSA: Diabetic rats fed with avocado-supplemented diet

Group DAE: Diabetic rats treated with aqueous mesocarp extract of avocado

To determine the effects of avocado on body weight, lipid profile and glycemic status and tolerance, rats in groups DC and DAE were fed with standard rat feed throughout the period of the study while DSA rats were fed with avocado-supplemented diet only. In addition, DAE rats were treated with avocado aqueous extract administered orally 
by orogastric cannular. The rats were monitored twice daily for food and water intake while body weight and FBG were assessed bi-weekly and recorded. The investigations using experimental animals were conducted in accordance with the internationally accepted principles for laboratory animal use and care [19] as found in the United States Guidelines (United States National Institutes for Health Publication No. 85-23, revised in 2011).

\section{Blood Collection and Biochemical Assay}

The blood samples were collected from both the tail veins (for OGTT) and cardiac puncture (for lipid profile). Samples from the tail veins were applied directly to the glucometer strips to determine the blood glucose concentration while those obtained through cardiac puncture were stored in $\mathrm{K}_{3}$ EDTA bottles for biochemical analysis.

\section{Glycemic Tolerance Assay}

Animals in all groups were fasted for 15 hours before the test with free access to water. Oral D-glucose load of $2 \mathrm{gm}$ per $\mathrm{kg}$ (dissolved in distilled water) was administered to the animals via flexible orogastric cannula. Thereafter, blood samples were withdrawn from the tail vein of each animal at 30 minutes interval for two-hour duration to determine blood glucose concentrations. Glycemic response curves were plotted using the blood glucose values obtained against time.

\section{Lipid Profile Assay}

The lipid profile analysis was conducted at the onset and 6 weeks after. Blood samples collected in the $\mathrm{k}_{3}$ EDTA (Ethylene Diamine Tetraacetic Acid) sample bottles were centrifuged at 3000 revolutions to obtain the plasma fractions which was kept in a refrigerator (at $-70^{\circ} \mathrm{C}$ ) until used and the sera obtained were used for the biochemical assay of the lipid profile. Plasma concentration of total cholesterol (TC), high density lipoprotein (HDL) and Triacylglycerol (TAG) were measured by the enzymatic colorimetric method after centrifugation using a drychemical automatic analyzer AU-5200 OLYMPUS (Randox Laboratories, San Francisco, USA). LDL level was determined by the Friedewald formula [20] as follows:

$\operatorname{VLDL}(\mathrm{mg} / \mathrm{dL})=\mathrm{TAG} / 5$

$\operatorname{LDL}(\mathrm{mg} / \mathrm{dL})=\mathrm{TC}-\mathrm{VLDL}-\mathrm{HDL}$

\section{Statistical Analysis}

Microsoft Excel and statistical SPSS program version 22 [21] were used for data analysis. Results are expressed as group mean \pm SEM. Comparison between groups were made using Students't-test and one way ANOVA. Significance level was set to $\mathrm{P}<0.05$.

\section{Results}

\section{Effect of Persea americana on Body Weight and Weight Gain}

The mean body weights at the onset and at the end of study for each animal group are shown in Table 2. Avocado consumption caused a significant decrease in mean body weight gain in both DSA $(13.75 \%)$ and DAE $(10.17 \%)$ rats compared with the diabetic control rats $(26.41 \%)$. The difference in the mean weight gain between the DSA and DAE groups was insignificant $(P>0.05)$. This study, demonstrated that avocado fruit mesocarp possesses beneficial weight-lowering potential which is more pronounced when consumed in the raw form than when supplemented in mixed meal.

\begin{tabular}{|l|l|l|l|}
\hline \multirow{2}{*}{ Parameters } & \multicolumn{3}{|l|}{ Diabetic Animal Categories } \\
\cline { 2 - 4 } & Control & $\begin{array}{l}\text { Avocado-Supplemented } \\
\text { Diet } \\
\text { DSA }\end{array}$ & Avocado Extract \\
& DC & $188.6 \pm 5.7$ & $179.8 \pm 17.9$ \\
\hline Final Mean Weight (g) & $210.6 \pm 14.8$ & $165.8 \pm 8.3$ & $163.2 \pm 11.7$ \\
\hline Initial Mean Weight (g) & $166.6 \pm 8.7$ & $13.75 \%$ & $10.17 \%$ \\
\hline$\%$ Mean Weight Gain & $26.41 \%$ &
\end{tabular}

Values are expressed in mean \pm SEM, Significant $(\mathrm{P}<0.05)$

Table 2: Effect of Avocado on Body Weight and Weight Gain $(n=5 /$ group $)$

\section{Effect of Persea americana on Glycemic Status}

The hypoglycemic effect of avocado on venous mean fasting blood glucose $(\mathrm{FBG})$ concentrations $(\mathrm{mg} / \mathrm{dL})$ in diabetic rats is depicted in Table 3. A significant $(P<0.05)$ reduction in mean FBG concentrations was observed in both DAE and DSA rats compared with the control. Difference in the mean FBG concentrations between DSA and DAE groups was comparably significant $(P=0.002)$. This observation revealed that raw (extract) avocado mesocarp impacts more hypoglycemic effect than avocado-supplemented diet.

\begin{tabular}{|c|c|c|c|}
\hline \multirow[t]{2}{*}{ Parameters } & \multicolumn{3}{|c|}{ Diabetic Animal Categories } \\
\hline & $\begin{array}{l}\text { Control } \\
\text { DC }\end{array}$ & $\begin{array}{l}\text { Avocado-Supplemented } \\
\text { Diet } \\
\text { DSO }\end{array}$ & $\begin{array}{l}\text { Avocado Extract- } \\
\text { DOE }\end{array}$ \\
\hline Final Mean FBG (mg/dL) & $160.6 \pm 15.7$ & $139.4 \pm 23.2$ & $120.4 \pm 5.0$ \\
\hline Initial Mean FBG (mg/dL) & $150.6 \pm 16.7$ & $154 \pm 32.3$ & $152.4 \pm 8.1$ \\
\hline$\%$ Change in Mean FBG & $6.64 \%$ & $-9.48 * \%$ & $-21.00 * * \%$ \\
\hline
\end{tabular}

Values are expressed in mean \pm SEM,

**Significant $(\mathrm{P}<0.05)$ when compared with DC and DSA.

*Significant when compared with DC.

Table 3: Effect of Avocado on venous mean FBG concentrations ( $m g / d L)$ $(n=5 /$ group $)$ 


\section{Effect of Persea americana on Glycemic Tolerance}

Effect of avocado on glycemic tolerance was assessed by the incremental areas under the glycemic response curves as depicted in Figure 1. Avocado significantly enhanced glycemic tolerance in DAE and
DSA rats compared with the control. The tolerance effect on DOE rats is comparably better and more improved over that of the DSO rats. The peak glycemic response to glucose load in all experimental groups occurred at 30 minutes of the 2 hour-duration

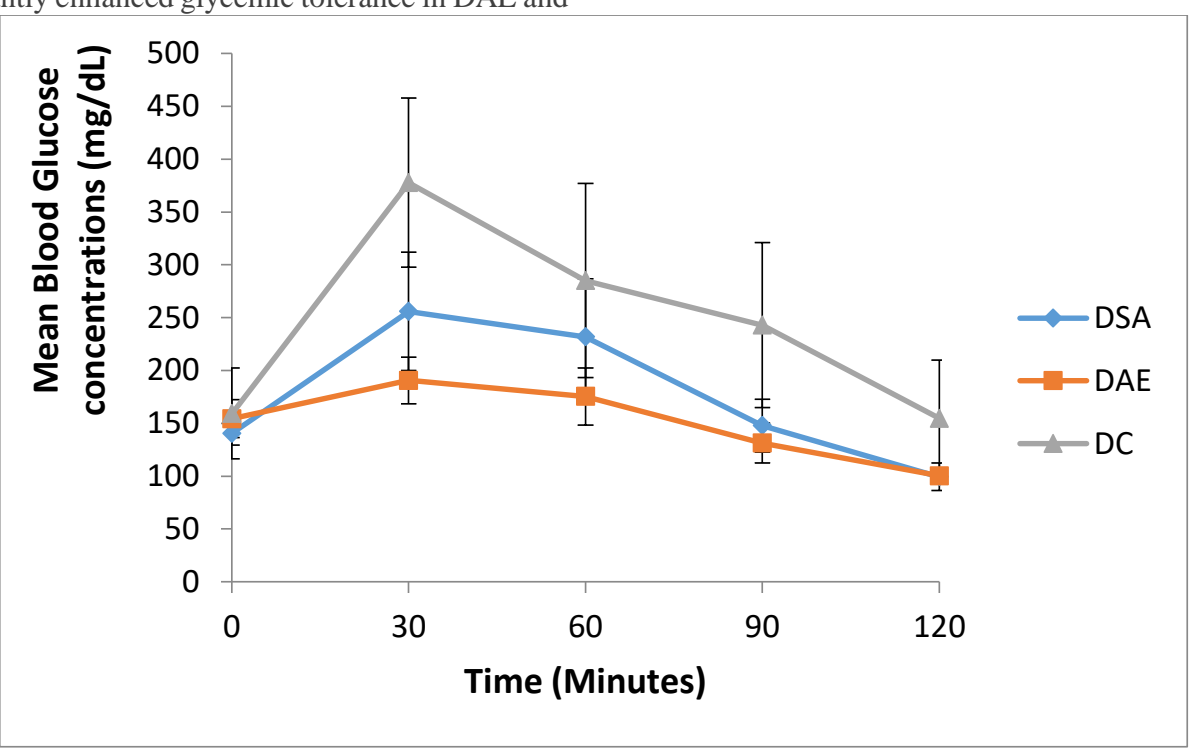

DC - Diabetic control rats,

DSA - Avocado supplemented diet-fed diabetic rats,

DAE - Avocado extract-treated diabetic rats.

Figure 1: Effect of avocado on glycemic tolerance profile ( $n=5 /$ group)

\section{Effect of Persea americana on lipid profile}

Figure 2 depicts the effect of avocado on lipid profile parameters in grouped experimental diabetic rats. After 6 weeks, avocado consumption caused significant decrease in TC, TG and LDL concentrations and a significant increase in HDL concentration in DSA and DAE rats compared with the control. In this study, avocado extract impacts more antilipidemic effect than avocado-supplemented diet.

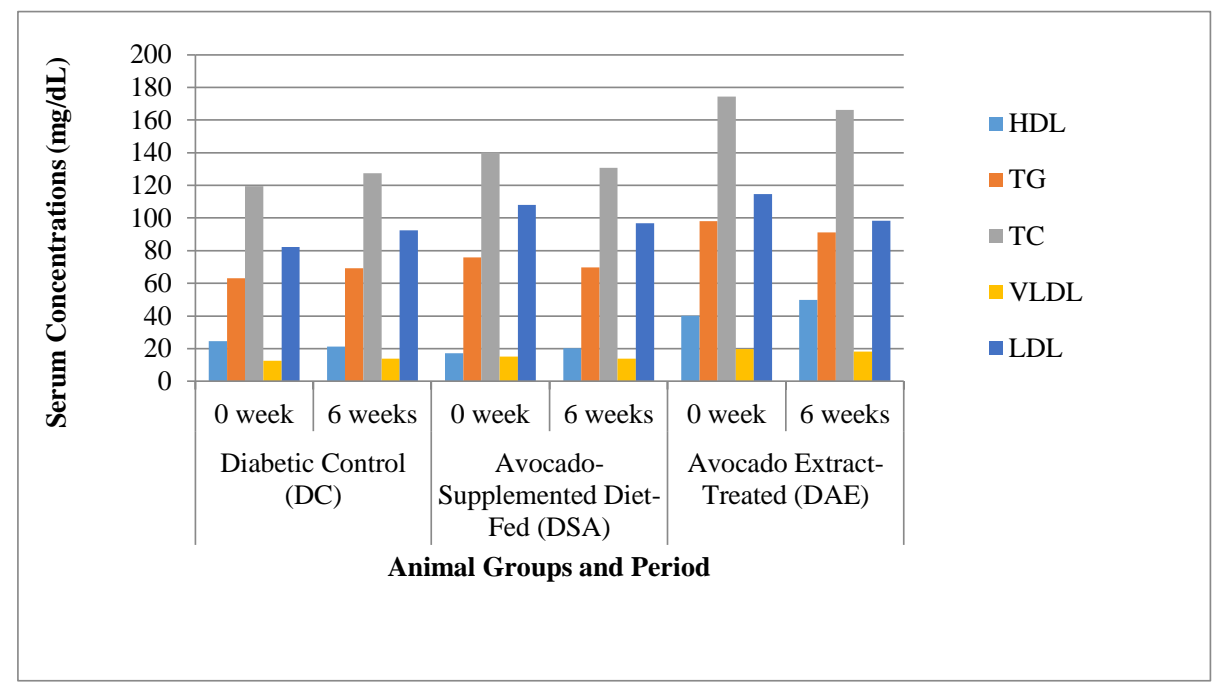

DC - Diabetic control rats, DSA - Avocado supplemented diet-fed diabetic rats,

DAE - Avocado extract-treated diabetic rats. TC: Total cholesterol;

TG: Triglycerides;

VLDL: Very Low-Density Lipoprotein; HDL: High Density Lipoprotein;

LDL: Low Density Lipoprotein. 


\section{Discussion}

Health benefits of avocado and the impacts of its mode of consumption on body weights, glycemic tolerance/control, and lipid profile in diabetic rats were investigated in this experimentally-controlled nutritional study which lasted for six weeks. Findings obtained revealed that avocado has beneficial antiobesity, hypoglycemic and antilipidemic potentials which are more expressed when consumed raw (fresh) than when ingested or supplemented in mixed meal.

Consumption of avocado in the raw (aqueous) and supplemented forms in this study significantly reduced weight gain in the experimental rats. However, aqueous extract of avocado demonstrated more weight-lowering effect than the avocado-supplemented mixed meal as shown in Table 2. This finding translates that eating avocado as fresh fruit confers adequate and optimal benefits in dietary management of weight reduction in diabetic condition than when consumed in mixed meals. Effect of avocado on body weight may be attributed to its high fibre content and low calories while presence of variety of vitamins, minerals and phytochemicals such as lutein, phenolic antioxidants, and phytosterols in avocado may also be contributing factors to this potential weight-reducing health benefits [22-24]. Various mechanisms related to the nutrients and bioactive compounds in avocados, may help to explain some of the findings related to weight changes which include impacts on satiety, metabolism, and gut microbiota. Avocado extract has been found to impact the expression of genes involved in fat metabolism and appetite in animals i.e., fatty acid synthase, fibroblast growth factor 21 , leptin and lipoprotein lipase [18].

On the glycemic status and profile, avocado consumption caused improved glycemic tolerance with significant reduction in mean FBG concentrations in DAE and DSA rats compared with the control. This finding agrees with the result of a study [18] which observed improvement in glycemic tolerance and insulin resistance in rats fed with high sucrose diets. In this study, difference in the mean values of FBG concentrations of DSA and DAE groups was comparably significant $(P=$ 0.002) which interprets that the hypoglycaemic effect of avocado was influenced by its mode of consumption in a similar manner to its effect on weight gain. While the glycemic response curves following glucose challenge peaked at 30 minutes in both DAE and DSA rats, the incremental areas under the glycemic response curves for DAE rats decreased more than that of DSA rats as shown in Figure 1. These results are promising, and more research is needed.

In people with type 2 diabetes, replacing some carbohydrate with avocado has been proved to help maintain blood glucose control [25]. Studies in healthy or overweight people have found either no compromise in blood glucose with avocado consumption [26,27] or significant reductions in postprandial glycemia after a half serve $(68 \mathrm{~g})$ or full serve (136g) of avocado [28]. Avocado contains monounsaturated fats that have been known to improve insulin sensitivity and lowers postprandial insulin $[29,30]$. Research studies using animal models have revealed mechanisms to explain these beneficial glycaemic effects of avocado. While some studies $[31,32]$ using avocado extracts showed that it modulates the activities of carbohydrate metabolic enzymes in rats, a study [33] observed that avocado extracts inhibit enzymes such as $\alpha$ amylase with reduction in oxidative stress in rat pancreas. Other studies $[34,35]$ reported increase in glucose uptake in the liver with normalization of liver enzyme levels.

Avocado in this study caused significant decrease in TC, TG and LDL-C concentrations with concomitant significant increase in HDL concentration in DAE and DSA rats (Figure 2). However, avocado extract impacts more beneficial antilipidemic effect than the avocadosupplemented diet. This finding therefore, suggests that the optimal effect of avocado is derived when consumed in fresh or raw form than in mixed meal. Several studies have shown that replacing some saturated fat in the diet with monounsaturated fat or polyunsaturated fat as richly contained in avocado can lead to health benefits. These benefits include increased insulin sensitivity, better blood sugar control and lower levels of LDL, total cholesterol and triglycerides [36]. A small randomized crossover study [26] using human subjects with type 2 diabetes fed with avocado diet for four weeks reported a decrease in cholesterol and triglycerides levels while another study [37] reported a significant reduction in total and LDL cholesterol in type 2 diabetic subjects fed with avocado for a week.

\section{Conclusion}

This study reveals that Persea americana fruit mesocarp possesses beneficial antiobesity, antidiabetic and antilipaemic potentials influenced significantly by its mode of consumption and preparation. For optimal effect, avocado is advised to be consumed raw or fresh rather than in mixed meals as revealed by the outcome of this study. Therefore, avocado consumption before meal especially should be encouraged and advocated in diabetic menu for normal weight, glycemic and lipid control.

\section{Authors Contributions}

This work was carried out in collaboration between the authors. Author MMCA designed, supervised, performed the analysis and interpretation of data and wrote the manuscript while author IAA assisted in the provision of essential materials and acquisition of data. Both authors read and approved the final manuscript for submission.

\section{Funding}

This research received no external funding.

\section{Conflicts of Interest}

The authors declare no conflict of interest.

\section{References}

1. Dreher, M.L. and Davenport, A.J. (2013). Hass avocado composition and potential health effects, Crit. Rev. Food Sci. Nutr. 2013, 53: 738-750.

2. Fulgoni 3rd, V.L., Dreher, M. and Davenport A.J. (2013). Avocado consumption is associated with better diet quality and nutrient intake, and lower metabolic syndrome risk in US adults: Results from the National Health and Nutrition Examination Survey (NHANES). Nutr. J. 2013, 12(1): 20012008.

3. Liu, X. J., Sievert J., Arpaia, M.L. and Madore, M.A. (2002). Postulated physiological roles of the seven-carbon sugars, mannoheptulose, and perseitol in avocado, J. Am. Soc. Hort. Sci. vol. 2002, 127: 108-114.

4. Paturi, G., Butts, C.A. and Bentley-Hewitt, K.L. (2017). Influence of Dietary Avocado on Gut Health in Rats, Plant Foods Hum. Nutr. 2017, 72: 321-323.

5. Wang, L. Bordi, P.L., Fleming,J.A., Hill, A.M. and KrisEtherton, P.M. (2015). Effect of a moderate fat diet with and without avocados on lipoprotein particle number, size and subclasses in overweight and obese adults: A randomized, controlled trial, J. Am. Heart Assoc. 2015, 4: e001355.

6. Mirmiran, P. et al., (2018). Fatty acid quality and quantity of diet and risk of type 2 diabetes in adults: Tehran Lipid and Glucose Study, J. Diabetes Complications. 2018, 32(7): 655659.

7. Qian, F. et al. (2016). Metabolic effects of monounsaturated fatty acid-enriched diets compared with carbohydrate or polyunsaturated fatty acid-enriched diets in patients with type 2 
diabetes: A systematic review and meta-analysis of randomized controlled trials, Diabetes Care. 2016, 39(8): 1448-1457.

8. Schwingshackl, L. et al. (2011): Effects of monounsaturated fatty acids on glycaemic control in patients with abnormal glucose metabolism: a systematic review and meta-analysis, Ann Nutr Metab. 2011, 58(4): 290-296.

9. Ryan, M. et al. (2000). Diabetes and the Mediterranean diet: a beneficial effect of oleic acid on insulin sensitivity, adipocyte glucose transport and endothelium-dependent vasoreactivity, QJM. 2000, 93(2): 85-91.

10. Shah, M. et al. (2007). Lipid, glycemic, and insulin responses to meals rich in saturated, cis-monounsaturated, and polyunsaturated (n-3 and n-6) fatty acids in subjects with type 2 diabetes, Diabetes Care. 2007, 30(12): 2993-2998.

11. Shaw, P.E. et al. (1980). High-performance liquid chromatographic analysis of D-manno-heptulose, perseitol, glucose, and fructose in avocado cultivars, J Agric Food Chem. 1980, 28(2): 397-462.

12. Leshem, B. et al. (1974). The hyperglycemic effect of 1-deoxyD-manno-heptulose. Inhibition of hexokinase, glucokinase, and insulin release in vitro, Can J Biochem. 1974, 52(11): 10781081.

13. Heskey, C., Oda, K. and Sabaté, J. (2019). Avocado intake, and longitudinal weight and body mass index changes in an adult cohort, Nutrients. 2019, 11(3): 691.

14. Pahua-Ramos, M.E. et al. (2014). Reduced-calorie avocado paste attenuates metabolic factors associated with a hypercholesterolemic-high fructose diet in rats, Plant Foods Hum. Nutr. 2014, 69:18-24.

15. Monika, P. and Geetha, A. (2015). The modulating effect of Persea americana fruit extract on the level of expression of fatty acid synthase complex, lipoprotein lipase, fibroblast growth factor-21 and leptin--A biochemical study in rats subjected to experimental hyperlipidemia and obesity, Phytomedicine. 2015, 22: 939-945.

16. Carranza-Madrigal, J., Herrera-Abarca, J.E., Alvizouri-Munoz, M., Alvarado-Jimenez, M.R. and Chavez-Carbajal, F. (1997). Effects of a vegetarian diet vs. a vegetarian diet enriched with avocado in hypercholesterolemic patients, 1997, Arch. Med. Res. 28: 537-541.

17. Pieterse, Z. et al. (2005). Substitution of high monounsaturated fatty acid avocado for mixed dietary fats during an energyrestricted diet: Effects on weight loss, serum lipids, fibrinogen, and vascular function, Nutrition. 2005, 21: 67-75.

18. Tabeshpour, J., Razavi, B.M. and Hosseinzadeh, H. (2017). Effects of Avocado (Persea americana) on Metabolic Syndrome: A Comprehensive Systematic Review, Phytother. Res. 2017, 31: 819-837.

19. National Research Council (US) Committee for the Update of the Guide for the Care and Use of Laboratory Animals (2011) Guide for the Care and Use of Laboratory Animals, National Academic Press, Washington DC, USA, $8{ }^{\text {th }}$ edition, 2011.

20. Friedewald, W.T., Levy, R.I. and Fredrickson, D.S. (1972). Estimation of the concentration of low-density lipoprotein cholesterol in plasma, without use of the preparative ultracentrifuge, Clin Chem 1072, 18: 499-502.

21. 'IBM SPSS Statistics for Windows, version 22 (IBM Corp., Armonk, N.Y., USA)'
22. USDA, Avocado, almond, pistachio, and walnut Composition, Nutrient Data Laboratory. USDA National Nutrient Database for Standard Reference, U.S. Department of Agriculture. Washington, DC, Release 24, 2011.

23. Unlu, N.Z., Bohn, T., Clinto, S.K. and Schwartz, S.J. (2005). Carotenoid absorption from salad and salsa by humans is enhanced by the addition of avocado or avocado oil, J. Nutr. 2005, 135: 431-436.

24. Wu, X. et al. (2004) Development of a database for total antioxidant capacity in foods: a preliminary study, J. Food Comp and Anal. 2004, 17: 407-422.

25. Del Toro-Equihua, M. et al. (2016). Effect of an avocado oilenhanced diet (Persea americana) on sucrose induced insulin resistance in Wistar rats, J. Food Drug Anal. 2016, 24(2): 350357.

26. Lerman-Garber, I. et al. (1994). Effect of a highmonounsaturated fat diet enriched with avocado in NIDDM patients," Diabetes Care. 1994, 17(4): 311-315.

27. Wien, M. et al. 2013). A randomized $3 \times 3$ crossover study to evaluate the effect of Hass avocado intake on post-ingestive satiety, glucose and insulin levels, and subsequent energy intake in overweight adults, Nutr J. 2013, 27(12): 155.

28. Mahmassani, H.A. et al. (2018). Avocado consumption and risk factors for heart disease: a systematic review and meta-analysis, Am J Clin Nutr. 107(4): 523-536.

29. Park, E. et al. (2018). Avocado Fruit on Postprandial Markers of Cardio-Metabolic Risk: A Randomized Controlled Dose Response Trial in Overweight and Obese Men and Women, Nutrients. 2018, 10(9): E1287.

30. Sabaté, J., Wien,M. and Haddad,E. (2015). Post-ingestive effects of avocados in meals on satiety and gastric hormone blood levels, Human Health Nut. 2015, 459-461.

31. Mahadeva Rao, U.S. et al. (2017). Salutary potential of ethanolic extract of avocado fruit on anomalous carbohydrate metabolic key enzymes in hepatic and renal tissues of hyperglycaemic albino rats, Chin J Integr Med. 2017.

32. Thenmozhi, A. et al. (2012). Biochemical evaluation of antidiabetic phytomolecule through bioactivity guided solvent fractionation and subfractionation from hydromethanolic (2:3) extract of Alligator pear Fruit in streptozotocin induced diabetic rats, J Appl Pharm Sci 2012, 2: 61.

33. Oboh, G. et al. (2014). Inhibition of key enzymes linked to type 2 diabetes and sodium nitroprusside induced lipid peroxidation in rats' pancreas by phenolic extracts of avocado pear leaves and fruit, Int J Biomed Sci. 2014, 10(3): 208-216.

34. Carvajal-Zarrabal, O. et al. (2014). Effect of dietary intake of avocado oil and olive oil on biochemical markers of liver function in sucrose-fed rats, Biomed Res Int. 2014.

35. Rao, U.S. and Adinew, B. (2011). Remnant B-cell-stimulative and anti-oxidative effects of Persea americana fruit extract studied in rats introduced into streptozotocin - induced hyperglycaemic state, Afr J Tradit Complement Altern Med. 2011, 8(3): 210-217.

36. Candido, F.G et al. (2017). Consumption of extra virgin olive oil improves body composition and blood pressure in women with excess body fat: A randomized, double-blinded, placebocontrolled clinical trial, Eur. J. Nutr. 2015, 57.

37. López Ledesma, R. et al. (1996). Monounsaturated fatty acid (avocado) rich diet for mild hypercholesterolemia, Arch Med Res. 1996, 27(4): 519-523. 
To Submit Your Article Click Here:

Submit Manuscript

DOI: $10.31579 / 2637-8914 / 071$
Ready to submit your research? Choose Auctores and benefit from:

$>$ fast, convenient online submission

$>$ rigorous peer review by experienced research in your field

$>$ rapid publication on acceptance

$>$ authors retain copyrights

$>$ unique DOI for all articles

$>$ immediate, unrestricted online access

At Auctores, research is always in progress.

Learn more https://auctoresonline.org/journals/nutrition-and-food-processing 